nämlich daß Euseb die Schrift mehrfach überarbeitet und erweitert hat, daß er auch fertige Stücke aus anderen seiner Werke oder aus unveröffentlichten eigenen Niederschriften und Sammlungen einbaute oder einfügen lie $\beta$, aber da $\beta$ er die notwendige Schlußredaktion nicht unternahm. Die Gründe kennen wir nicht, doch liegt die Annahme nahe, daß er vor der letzten Redaktion starb und das Buch deshalb postum erschien. Auch störende Stellen im Text, die wie spätere Randglossen aussehen und in dieser Edition in Klammern gesetzt sind, könnten also durchaus auf Euseb selbst zurückgehen, der sie anfügte, aber nicht mehr richtig einarbeiten konnte (z. B. S. 15, 4; 17, 11; $18,7)$. Die Beurteilung wird durch die mangelnde Qualität der Überlieferung der VC (siehe oben S. XXV), die man immer mit in Rechnung stellen muß, erschwert.

Aus dem nächsten Kapitel ergibt sich, daß der Stil der VC nicht gleichmäßig ist, daß man also auch von den sprachlichen Indizien aus weder Interpolationen konstatieren noch andererseits die Echtheit begründen kann. Philologische Mittel können nicht entscheidend zur Lösung der Authentizitätsfrage der VC beitragen, sondern spielen dabei nur eine mittelbare Rolle. Doch ist $\mathrm{m}$. E. weder der inhaltliche noch der philologische Tatbestand mit der Annahme der Echtheit der VC unvereinbar. Wichtiger als die Frage der Authentizität der Schrift ist aber die nach der Glaubwürdigkeit, die schon in der Authentizitätsdebatte eine Rolle spielte. Ihre Beantwortung führt über die Aufgabenstellung einer Edition hinaus.

\title{
Sprache und Stil ${ }^{1}$
}

Der gewählten Gattung der Schrift wie der Größe des behandelten Gegenstandes entsprechend, bemüht sich Euseb um eine elegante, gehobene Sprache, nämlich um die Erfüllung der attischen Norm, um die Wahl älterer, poetischer Begriffe ${ }^{2}$, um ein uns etwas manieriert

1 Die Arbeit von E. Fritze (Beiträge zur sprachlich-stilistischen Würdigung des Eusebios, Phil. Diss. München 1909, gedruckt Borna-Leipzig 1910) reicht zur Beurteilung der VC nicht aus. Einmal wirkt sich nachteilig aus, daß er Hist. Eccl. I-V, VC und Laus zusammen auswertet, obwohl die Schriften nicht der gleichen Gattung angehören. So ergibt sich ein verwaschenes Bild. Zum anderen fußt er vor allem auf Heikels Text und beachtet nicht genügend die von Heikel verworfenen Lesarten. Fritze hätte durch seine Arbeit zu einer umfassenderen Kritik der Ausgabe Heikels kommen können. So manche Widersprüche des Sprachgebrauches der VC, die Fritze feststellte, beruhen lediglich auf der Textwahl Heikels.

Der Sprachgebrauch der VC weicht oftmals von dem der Hist. Eccl. ab. Doch spricht das nicht gegen eine eusebianische Abfassung der VC. Denn die VC will in Stil und Form gepflegter sein als andere Werke Eusebs.

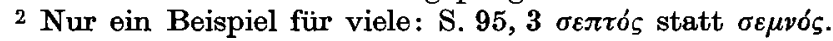


anmutendes Griechisch. Die Sprache der VC ist aber keineswegs einheitlich, denn sie bietet auch eindeutig bezeugt Koinegriechisch, das nicht etwa auf spätere Eingriffe in den Text zurückzuführen ist. Euseb ist kein reiner Stilist, sondern auch ein Mann der kirchlichen Praxis, bei dem sich der Einfluß des Studiums von Bibel und Väterliteratur und des kirchlichen Umgangsgriechisch bemerkbar macht, und ein Materialsammler, dem - wenn man auf die Gesamtheit seiner Schriften sieht - der Inhalt mehr wert ist als die äußere Form. Einige Ungleichheiten im Stil verwundern allerdings trotzdem 1, doch zu ihrer Erklärung reicht die Annahme aus, daß die letzte glättende Hand Eusebs am Text fehlt. Dazu kommt, daß unsere Überlieferung schlecht ist und spätere vulgäre Sprachelemente den ursprünglichen Text an einigen Stellen verdorben haben können.

\section{Zur Formenlehre}

Bei den Konsonantenverbindungen ist die attische Form bevorzugt,

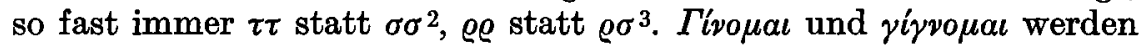
durcheinander gebraucht, doch nur $\gamma \iota v \omega \sigma x \omega$. Neben dem attischen $\sigma \mu \iota x \varrho o ́ s ~ s t e h t ~ s e h r$ oft $\mu \iota \varkappa o_{\varsigma}{ }^{4}$. Verhältnismäßig oft begegnet das von den Attizisten vermiedene $\mu \eta \vartheta \vartheta_{\varepsilon} \nu^{5}$. Der Hiatus wird nach Möglichkeit

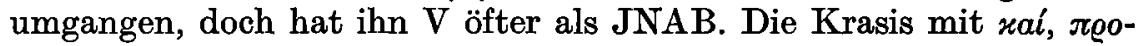
oder dem Artikel ist sehr beliebt 6 .

Als Contracta der 2. Deklination sind zu nennen: ỏotéa (S. 110, 22),

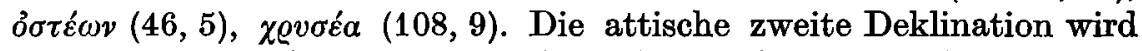
gern gebraucht: Fast immer veẃs und seine Formen $^{7}$; dagegen fast immer $\lambda \alpha o^{\prime} \varsigma$ statt $\lambda \varepsilon \omega^{\prime} \varsigma^{8} ; \tilde{\varepsilon} \omega \varsigma$ und seine Formen ${ }^{9} ; \not \dot{\alpha} \gamma \eta \dot{\jmath} \omega^{10}$. Bei der 3. Deklination ist zu erwähnen, daß neben dem Gen. $\alpha$ ó $\tau \varepsilon \omega \varsigma$ der vul-

1 Zum Beispiel werden manchmal zwei verschiedene Formen desselben Wortes in einem Zusammenhang gebraucht.

${ }^{2} \sigma \sigma$ nur S. 49,15; 83, 24 (in unattischen Wörtern); 100, 20; 134,23 S. 82, 18 steht in einem LXX-Zitat.

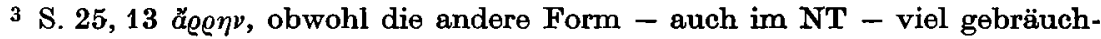
licher war. V bevorzugt falsch öfter $\dot{\varepsilon} \pi \imath \vartheta \varrho \varrho \sigma \varepsilon \omega$. Eindeutig $\varrho \sigma$ nur S. 132, 18

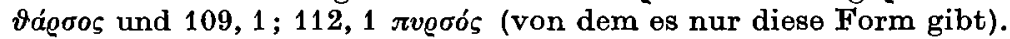

$4 \mathrm{~V}$ bevorzugt $\sigma \mu \iota x \varrho o ́ s$. Mıx@ós eindeutig: S. 15, 20;18,9; 54,20; 54, 27; 66,$14 ; 72,29 ; 80,5 ; 102,2 ; 112,2$.

5 S. 22,$25 ; 28,23 ; 29,21 ; 44,9 ; 48,22 ; 53,1 ; 127,24 ; 135,11$.

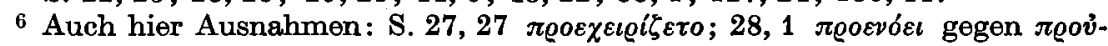

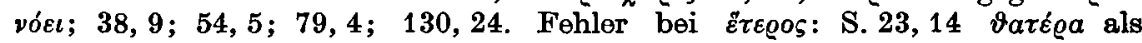
Nom. fem. sing. und S. 41, 4 Vatẓ́as als Gen. fem. sing.

7 vaós nur S. 103, 2; 126, 2.

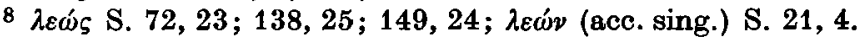

9 S. 15,$12 ; 128,11 ; 130,18$; schlecht S. 130,18 éwas JNAB.

10 S. 19,$15 ; 148,19$. 


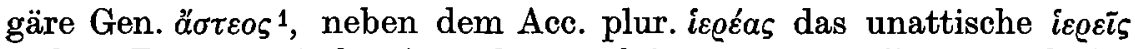
steht ${ }^{2}$. Der unattische Acc. plur. auch in pa $\mu a x \varepsilon \bar{i}^{3}{ }^{3}$. Dagegen findet

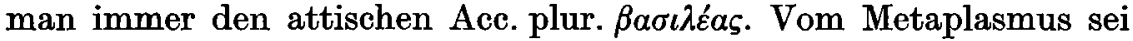

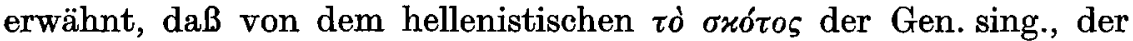

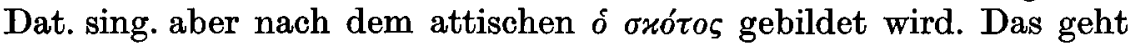
so weit, daß Euseb - gegen seine sonstige Geptlogenheit in der VC -

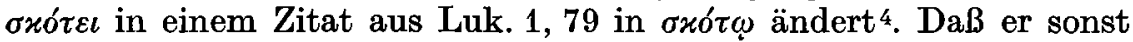
Bibelzitate nicht umändert, sei an einem Beispiel demonstriert: der

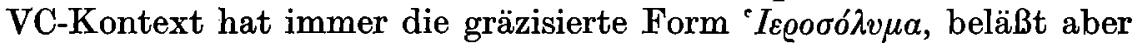

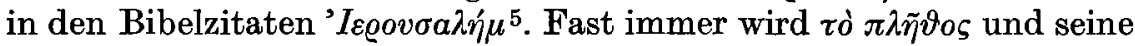
Formen, nur selten $\dot{\eta} \pi \lambda \eta \vartheta v^{\prime}$ gebraucht ${ }^{6}$. Bei der Deklination der unregelmäßigen Komparationsformen stehen kontrahierte und nichtkontrahierte Formen nebeneinander: Nur nichtkontrahierter Acc. sing.

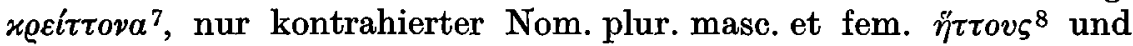
$\pi \dot{\lambda}$ íov $^{9}$, nur kontrahierter Acc. plur. masc. $\pi \lambda$ cíov $^{10}$, Nom. et Acc.

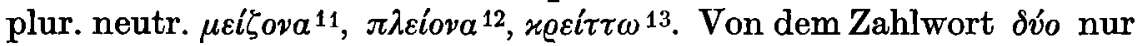
die hellenistische Flexion 14. Der Dual wird kaum noch gebraucht ${ }^{15}$.

Bei den Verba contracta werden die kontrahierten Formen ge-

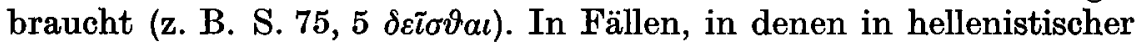
Zeit (auch im NT) der Aor. II aufkommt oder beliebt und gängig ist, bemüht sich die VC um den Aor. I ${ }^{16}$. Charakteristisch für die attizistischen Tendenzen der VC ist auch die starke Hervorhebung der äolischen Formen des Opt. aor. act. ${ }^{17}$. In der zweiten Konjugation ge-

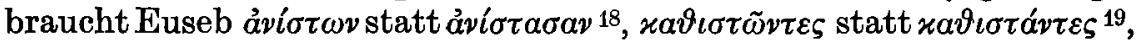

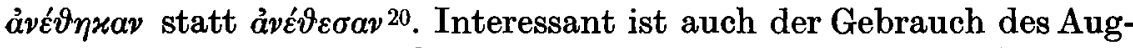
ments: nur einmal wird das Plusquamperf. ( $\tau \alpha \dot{\tau} \tau \omega)$ mit Augment gebildet ${ }^{21}$ gegen dreizehnmal ohne Augment ${ }^{22}$. Stets fehlt das Augment

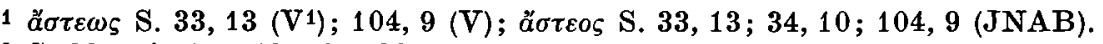

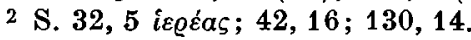

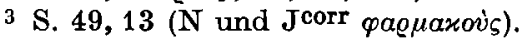

4 S. 56, 1. In NT und LXX die hellenistische Form. ó бxótos nur in Hebr. 12, 8.

5 S. 99, 14. 18 nur dieser Begriff aus dem NT.

6 i $\pi \lambda \eta \vartheta \vartheta^{\prime} \varsigma$ nur S. 17,$17 ; 24,27 ; 81,25 ; 85,13$.

7 S. 27,$1 ; 53,6 ; 103,6$.

8 S. 51,$18 ; 81,16$.

9 S. $52,21(\mathrm{~V}) ; 54,28 ; 111,19 ; 120,12 ; 135,6$.

10 S. 66,$19 ; 122,21 . \quad 11$ S. $16,25 . \quad 12$ S. $43,27$.

13 S. $143,20$.

14 S. 18,$5 ; 57,32 ; 66,24 ; 101,22 ; 102,13 ; 102,14$. Dat. $\delta v \sigma i$ S. 41, 2; $101,7$.

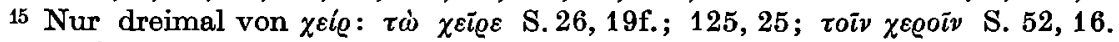
$a ̈ \mu \varphi \omega$ wird mit dem Plural gebraucht.

16 Nur ein Beispiel für sehr viele: S. 81,1 $\varphi \lambda \varepsilon \chi \vartheta \dot{v} \nu \tau a$ V statt $\varphi \lambda \varepsilon \gamma \varepsilon \dot{v} \tau a$ JNB.

17 S. 15,$11 ; 32,15 ; 39,17 ; 49,15 ; 50,13 ; 50,20 ; 95,22 ; 120,16 ; 130,19$; 148,14

18 S. 53, 26. $\quad 19$ S. 148, $23 . \quad 20$ S. $20,1.21$ S. $85,21$.

${ }_{22}$ S. 22,$22 ; 24,19 ; 34,12 ; 40,7 ; 48,1 ; 52,2$ (JNAB gegen V); 53,13 ; 55,$13 ; 57,27 ; 66,15 ; 94,7 ; 95,13 ; 112,18$. 
bei $\varepsilon \dot{v} \varrho i \sigma x \omega$ und $x \dot{q} \vartheta \eta \mu a \imath$. Nebeneinander gebraucht werden z. B.

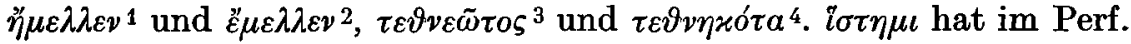
nur die Kurzformen.

\section{Zur Syntax}

Auch hier sollen nur besonders charakteristische Punkte hervorgehoben werden. Zu erwähnen ist der öfter anzutreffende Dat. sociat. bei avं ós ${ }^{5}$. Eine weitere attizistische Tendenz zeigt sich in dem Dativ + Passiv statt vijó ( $\pi \varrho o ́ s)+$ Gen. ${ }^{6}$. Unklassische Einflüsse zeigt dagegen der temporale Dativ auf die Frage „wie lange?" 7 .

Charakteristisch für den Attizismus der VC ist das Zurücktreten des Konjunktivs und das Aufleben des vom hellenistischen Griechisch verdrängten Optativs. „Obwohl also bei Eusebios der Optativ zuungunsten des Konjunktivs im Vordringen ist, bleibt doch der Optativgebrauch an und für sich ein beschränkter, ja sein Rückgang ist unverkennbar ... Die künstliche Belebung und mehr dekorative Verwendung des Optativs bei Eusebios ist deutlich erkennbar" 8 . Hervorzuheben ist der Opt. potent. ${ }^{9}$ und der Opt. obliquus, der nach Praes., Praeter. und Fut. im Hauptsatz eintritt und vielfach den Konjunktiv ersetzt ${ }^{10}$.

Sehr oft findet man das näher bestimmende Partizip beim Verbum

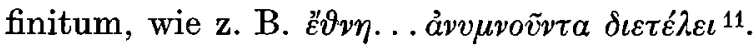

In den Konsekutivsätzen hat die VC eine Vorliebe für die Konjunktion $\hat{\omega} \varsigma$ + Infinitiv ${ }^{12}$, in den Finalsätzen für $\dot{\omega} \varsigma \alpha \ddot{v}+$ Optativ ${ }^{13}$.

In der Frage des Gebrauches der beiden Negationen, des objektiven $o v$ und des subjektiven $\mu \eta^{\prime}$, vertritt Euseb natürlich nicht mehr die Kompliziertheit der klassischen Sprache. Bei ihm sind schon starke Einflüsse der Volkssprache zu konstatieren. Auch von der Koine des NT weicht er hier ab ${ }^{14}$. Nach den Ausdrücken des Hinderns (ả $\pi \varepsilon \ell \varrho \ell \varepsilon \iota v$,

1 S. $93,8 . \quad 2$ S. 25,$15 ; 110,20$.

3 S. $27,13$.

4 S. 27,15 .

5 S. 22,$4 ; 24,25$ u. a.

6 S. 20,$19 ; 50,1$ u. v. a.

7 S. 24,$19 ; 83,6 ; 107,30 ; 108,7 ; 110,9 f . ; 119,20$. Über die Gründe dieses Dativs vgl. F. Blass - A. Debrunner, Grammatik des neutestamentlichen Griechisch, 7. Aufl. Göttingen 1943, § 201.

8 Fritze, a. a. O. 18. Hier sind auch Beispiele aufgeführt.

9 S. 19,$18 ; 19,24 ; 20,4 ;$ u. v. a.

10 Zum Beispiel Finalsätze, s. S. 18, 11

11 S. $37,8.11$ u. a.

12 S. $16,14 f$.; $17,27 f$. u. a.

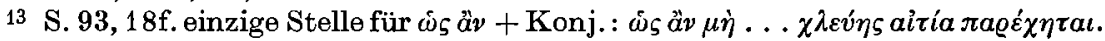

14 Hier gilt die Regel: „ov̉ negiert den Indikativ, $\mu \eta \dot{~ d i e ~ u ̈ b r i g e n ~ M o d i ~ e i n-~}$ schließlich Infinitiv und Partizipium" (Blass-Debrunner, a. a. O. § 426). 


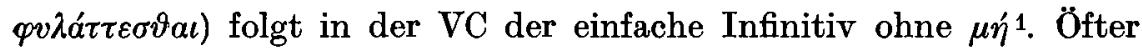
tritt $\mu \eta^{\prime}$ für $o v$ ein ${ }^{2}$. Erwähnt werden soll noch die Vorliebe für die Häufung der Negationen, z. B. $\mu \eta \delta \alpha \mu \tilde{\eta} \mu \eta \delta \alpha \mu \tilde{\omega} \varsigma^{3}$.

Bei den Präpositionen ${ }^{4}$ bemüht sich die VC, sich gegen die Tendenz der Sprachentwicklung zu stemmen und sich der Klassik zu nähern. Der Dativ ist zwar im Abnehmen begriffen, doch wird er vielfach noch richtig angewendet. Auch $\dot{\varepsilon} v$ wird noch sehr oft gebraucht und noch

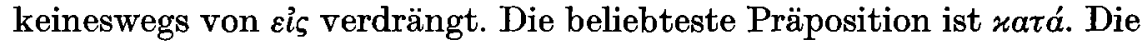
klassische Tendenz zeigt sich deutlich in dem Wiederaufleben der Präposition $\dot{\alpha} \mu \varphi \dot{i}$, ja sogar an mehreren Stellen $\dot{\alpha} \mu \varphi \dot{i}+$ Gen. ${ }^{5}$, und der Präposition ảvó.

\section{Zum Stil6}

Der Stil ist breit, überladen, gesucht kompliziert und gespreizt.

Sehr ins Auge fällt die Häufung der Synonyma. Nur einige wenige

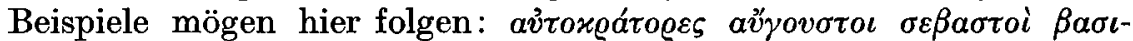

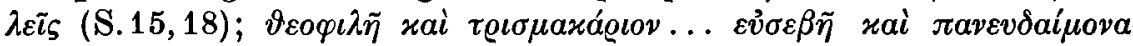

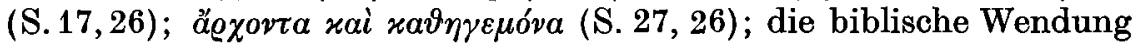

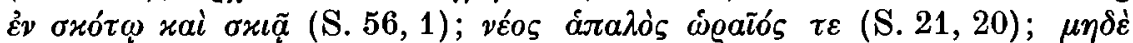

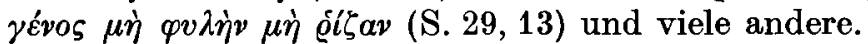

Ebenso ist in erstaunlichem Ausmaß die Alliteration und die Annomination gebraucht. Auch hier nur einige Beispiele aus der Fülle:

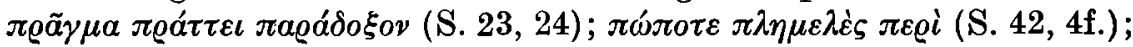

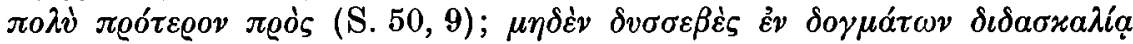

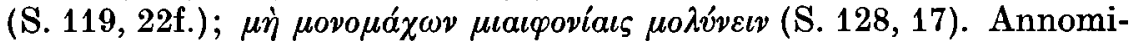

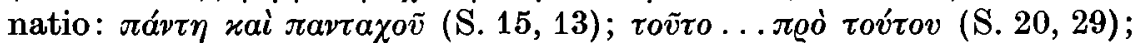

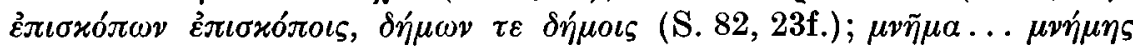

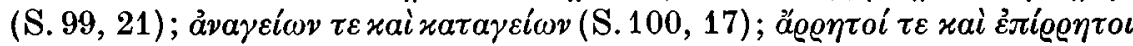

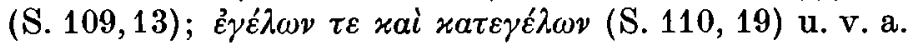

Da $B$ auch viele Fälle von Pleonasmus registriert werden können,

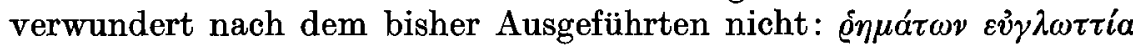

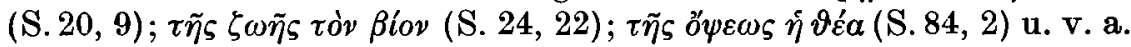

Attizistische Tendenz zeigt die VC in der großen Vorliebe für Ellipsen von Substantiven oder von allen Formen des Verbums eiv $\boldsymbol{l}^{7}$.

1 S. 26,$11 ; 126,2$.

2 Zum Beispiel S. 24, 7; 20, 29

S. 22,$6 ; 42,8 ; 81,3$.

4 Vgl. dazu die statistischen Aufstellungen bei Fritze, a. a. O. 26-32 ; allerdings sind sie nicht für die VC getrennt aufgeführt.

5 S. 22,$20 ; 72,21 ; 104,3 ; 132,16.19$.

6 Für die Redefiguren vgl. Fritze, a. a. O. $36 \mathrm{ff}$.

7 Einige Beispiele: 18, 10; 37, 14; 56, 24; 102, 29; 126, 22; 128, 14. Für eivaı sind die Fälle so häufig, daß ich hier keine Beispiele anführe. Das hatte Heikel in seiner Ausgabe nicht genügend beachtet. Manche seiner Konjekturen sind deshalb unnötig. 
Die gleiche Tendenz tritt in dem verbreiteten Gebrauch des Prinzips

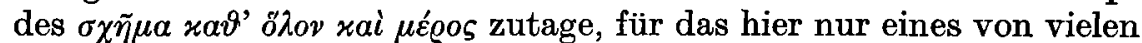

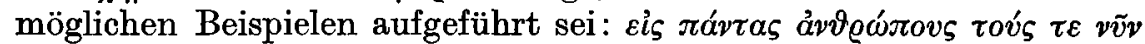

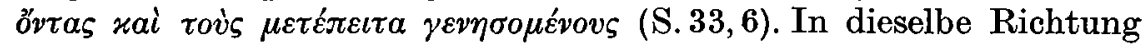
gehört die Verwendung der Distributio. Auch hier seien aus der Fülle

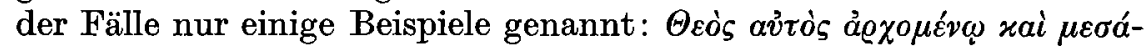

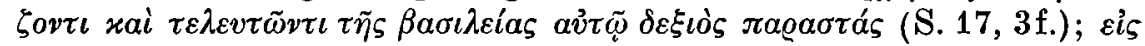

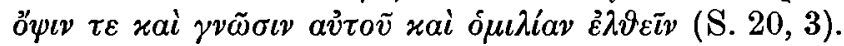

Die besondere Betonung des oratorischen Elementes wird zum Beispiel auch in der Verwendung der rhetorischen Frage deutlich ${ }^{1}$. Hier sei als Beispiel nur die interessante Stelle S. 121, 10 genannt: $\tau \dot{\imath} \delta \varepsilon$

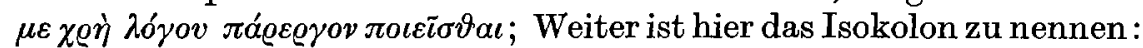

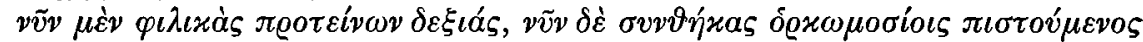
(S. 41, 24). Ebensoviel Wert ist auf die Antithesen gelegt: ă $\varrho \tau \iota \mu \dot{\varepsilon} \nu .$.

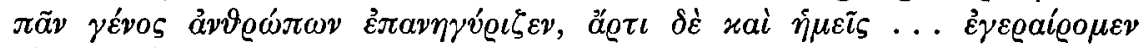

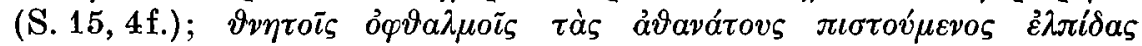
(S. 16, 27f.) und sehr viele andere.

Ins Auge fallen die langen Perioden, die durch $\mu \dot{\varepsilon} v-\delta \dot{\varepsilon}$ oder $\tau \varepsilon-\tau \varepsilon$ u. a. gebildet werden. In dem Zusammenhang sei auch die große Vor-

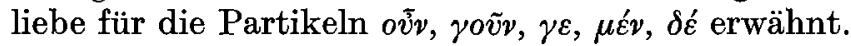

Das Künstliche des VC-Stiles kommt auch in der Wortstellung zum Ausdruck. Hier will der Verfasser nach Möglichkeit das Geläufige und Übliche umgehen, in einer Weise jedoch, daß sich keine Regeln aufstellen lassen. Vor allem übt die VC die Umstellung des Verbums an einen ungewöhnlichen Ort, so daß eng Zusammengehörendes (Worte oder Sätze) auseinandergerissen wird. Der Leser wird bei der Lektüre ständig auf Beispiele stoßen. Erwähnt sei noch, daß die VC neben der richtigen Stellung der Gattungsbezeichnung auch solche Fälle

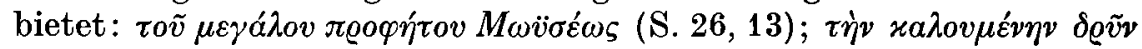
$M \alpha \mu \beta \varrho \tilde{\eta}(\mathrm{S} .105,13)$ u. a.

Zur Bestimmung der richtigen Wortstellung bei Differenzen der Handschriften hilft auch nicht die Feststellung einer gewissen Vorliebe für bestimmte Formen des akzentuierten Satzschlusses, weil sich die VC noch am Anfang einer Entwicklung befindet und in ihr noch keine durchgehende Methode waltet. Der Beweis, daß die VC schon bestimmte Satzschlüsse bevorzugt, sei durch das Material des ersten Buches erbracht 2:

Form 0 (keine unbetonte Silbe vor dem letzten Akzent)

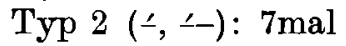

Typ $3(\llcorner,-1)$ : 4 mal

1 S. 19,$28 ; 24,8 ; 30,3$ u. v. a.

2 Ich halte mich dabei an das Formen- und Typenschema, das Stanislas Skimina, État actuel des études sur le rhythme de la prose grecque II, Lwow 1930, 9 (Eus Supplementa 11) bietet. 
Form I (eine unbetonte Silbe vor dem letzten Akzent)

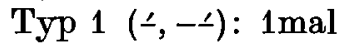

Typ $3(\stackrel{2}{\prime},-\infty)$ : $6 \mathrm{mal}$

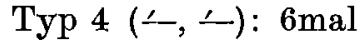

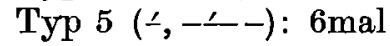

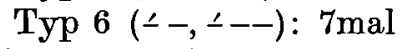

Form II (zwei unbetonte Silben vor dem letzten Akzent)

Typ $1(\leftarrow,--\not)$ : 2mal

Typ $2(\llcorner,-<): 11 \mathrm{mal}$

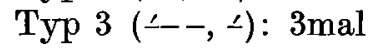

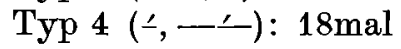

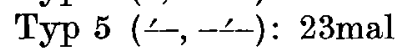

Typ 6 (

Typ $7\left(\leftarrow,--\_-\right)$: 9mal

Typ $8(\dot{-},-,--)$ : $20 \mathrm{mal}$

Form III (drei unbetonte Silben vor dem letzten Akzent)

Ohne Typenaufschlüsselung: $56 \mathrm{mal}$

44 Fälle mit vier und mehr unbetonten Silben vor dem letzten Akzent.

Aus dem Material ist zu ersehen, daß Form II - Typ 9 fehlt aber sehr bevorzugt wird. Es folgt dann Form III. Für die Verteilung der einzelnen Formen läßt sich kein Schema aufstellen. Daß man aus dem Abweichen von den beliebten Formen keine Schlüsse auf die Unechtheit der betreffenden Stellen ziehen darf, braucht kaum hervorgehoben zu werden.

Ein System quantitierender Klauseln habe ich daneben in der VC nicht finden können. $\mathrm{Ab}$ und an begegnet eine der in hellenistischer Zeit beliebten Formen ${ }^{1}$, doch liegt m. E. keine Methode vor. I. Heikel hielt den ganzen VC-Kontext für mehr oder weniger rhythmisiert: „Wer sein Ohr an den Klang ,antiker Kunstprosa' etwas gewöhnt hat, wird nicht viele Zeilen aus der Vita Constantini lesen können, ohne daß ihm ... der rhetorische Ton stark auffällt. Ein gewisser Unterschied zwischen den einzelnen Partien kommt freilich vor, aber alles, was von Eusebius selbst herrührt, ist in rhythmischer Sprache abgefaßt. Bekanntlich ist es gar nichts Leichtes, diesen numerus orationis systematisch vorzuführen, er muß vor allem gefühlt werden ... In den Partien ruhigen erzählenden Inhalts ist der Rhythmus natürlich weniger ausgeprägt . . . aber der Grundcharakter des Ganzen ist rhythmisch“2. Im Anfang (S. 15, 4-9) fällt der daktylische Klang auf. In der übrigen Schrift begegnet dann und wann einmal ein metrisches Maß. Eine bewußte rhythmisierende Methode läßt sich aber kaum

1 Siehe A. W. de Groot, Der antike Prosarhythmus I, Groningen, Haag 1921, 62ff. 105 und ders., La prose métrique des anciens, Paris 1926, $32 \mathrm{ff}$. (Collection d'études latines II).

2 GCS 7, LIf. 
feststellen. Besonders rhetorisch und in gespreizter Ausdrucksweise sind das Prooemium und die ersten Seiten des 3. Buches, im Unterschied zum übrigen Text, abgefaßt.

Ein einziges griechisches Urteil über den Stil der VC ist uns erhalten, das des in Stilfragen sehr strengen Photius, der in Cod. 127 der Bibliotheca sagt, der Stil der VC gleiche dem der anderen Euseb-

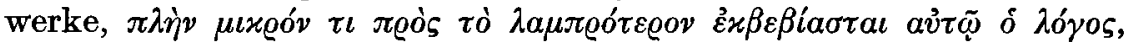

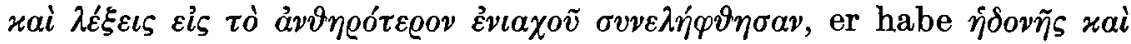

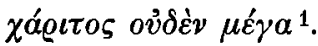

Die Sprache der konstantinischen Urkunden hat I. A. Heikel gründlich untersucht ${ }^{2}$. Auf seine Ausführungen sei hier verwiesen.

\section{Zur Textausgabe}

Bei der Textkonstituierung wurde behutsam vorgegangen. Der von den Textzeugen gebotene Text ist also nur in unumgänglichen Fällen geändert. Solange eine von den Handschriften gebotene Lesart verständlich ist, wurde sie belassen ${ }^{3}$.

Keine Handschrift der direkten Überlieferung ist so gut, daß man ihr unbesehen folgen könnte 4 . Es ließen sich also keine ganz sicheren und durchgängig anwendbaren Gesetze für die Textbildung aufstellen. Ausschlaggebend bei der Textwahl waren die folgenden Gesichtspunkte:

Allgemein: a) Stützung durch andere gute Zeugen ( $a$, Papyrus, Sokrates, Sozomenos, Theodoret, hagiographische Viten).

VC-Kontext: b) Übereinstimmung mit dem Stil der VC. Lesarten, die attizistische Gewohnheiten vertreten, sind also bevorzugt. $\mathrm{Zu}$ beachten ist allerdings, daß der Stil der VC nicht gleichmäßig ist.

1 Photios, Bibl., ed. R. Henry, II, Paris 1960, 100.

2 GCS 7, Leipzig 1902, LXXII-LXXXII.

3 Fast auf jeder Seite begegnen ein oder mehrere Fälle, in denen Konjekturvorschläge von Valesius, Wilamowitz, Heikel u. a. nur im Apparat vermerkt sind, da das Griechisch der klassischen Periode nicht als Maßstab für die VC gelten kann.

Weil die VC postum erschien und die letzte Hand Eusebs am Text fehlt, ist es m. E. nicht richtig, aus Gründen der Gleichförmigkeit bei Stilunterschieden u. ä. gegen das Zeugnis der Handschriften Eingriffe vorzunehmen. Dafür seien einige wenige Beispiele angeführt: S. 101,9 ist von allen Hand-

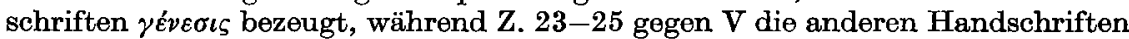

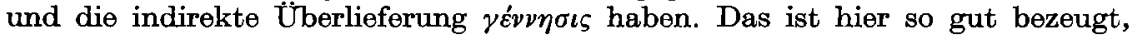
daß man am besten beide Formen auf einer Seite nebeneinander stehen läßt. $V$ steht im Verdacht der Vereinheitlichung. S. 149, 10 haben fast alle Hand-

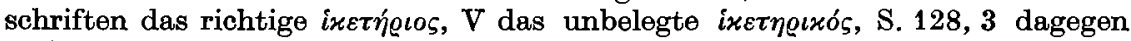

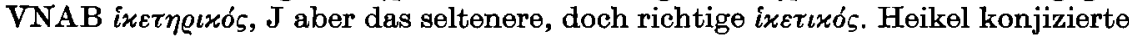

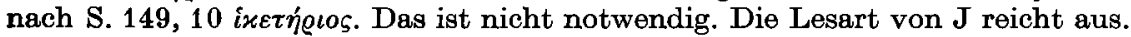

4 Vgl. dazu oben S. XXV-XXVII. 